\title{
The Gaussian measure on algebraic varieties
}

\author{
by
}

Ilka Agricola and Thomas Friedrich (Berlin)

\footnotetext{
Abstract. We prove that the ring $\mathbb{R}[M]$ of all polynomials defined on a real algebraic variety $M \subset \mathbb{R}^{n}$ is dense in the Hilbert space $L^{2}\left(M, e^{-|x|^{2}} d \mu\right)$, where $d \mu$ denotes the volume form of $M$ and $d \nu=e^{-|x|^{2}} d \mu$ the Gaussian measure on $M$.
}

1. Introduction. The aim of the present note is to prove that the ring $\mathbb{R}[M]$ of all polynomials defined on a real algebraic variety $M \subset \mathbb{R}^{n}$ is dense in the Hilbert space $L^{2}\left(M, e^{-|x|^{2}} d \mu\right)$, where $d \mu$ denotes the volume form of $M$ and $d \nu=e^{-|x|^{2}} d \mu$ is the Gaussian measure on $M$. For $M=\mathbb{R}^{n}$, the result is well known since the Hermite polynomials constitute a complete orthonormal basis of $L^{2}\left(\mathbb{R}^{n}, e^{-|x|^{2}} d \mu\right)$.

2. The volume growth of an algebraic variety and some consequences. We consider a smooth algebraic variety $M \subset \mathbb{R}^{n}$ of dimension $d$ and denote by $d \mu$ its volume form. Then $M$ has polynomial volume growth: there exists a constant $C$ depending only on the degrees of the polynomials defining $M$ such that for any euclidian ball $B_{r}$ with center $0 \in \mathbb{R}^{n}$ and radius $r>0$ the inequality

$$
\operatorname{vol}_{d}\left(M \cap B_{r}\right) \leq C r^{d}
$$

holds (see [Brö]). Via the Crofton formulas the above inequality is a consequence of Milnor's results concerning the Betti numbers of an algebraic variety (see [Mi1], [Mi2], in which the stated inequality is already implicitly contained). This estimate yields first of all that the restrictions to $M$ of polynomials on $\mathbb{R}^{n}$ are square-integrable with respect to the Gaussian measure on $M$.

1991 Mathematics Subject Classification: 28A75, 58A07, 14P99.

Key words and phrases: Gaussian measure, algebraic variety.

The work was supported by the SFB 288 "Differential geometry and quantum physics". 
Proposition 1. Let $M$ be a smooth submanifold of the euclidian space $\mathbb{R}^{n}$. Suppose that $M$ has polynomial volume growth, i.e., there exist constants $C$ and $l \in \mathbb{N}$ such that for any ball $B_{r}$,

$$
\operatorname{vol}_{d}\left(M \cap B_{r}\right) \leq C r^{l} .
$$

Then:

1. The ring $\mathbb{R}[M]$ of all polynomials on $M$ is contained in the Hilbert space $L^{2}\left(M, e^{-|x|^{2}} d \mu\right)$.

2. The functions $e^{\alpha|x|^{2}}$ for $\alpha<1 / 2$ all belong to $L^{2}\left(M, e^{-|x|^{2}} d \mu\right)$.

Pr o of. Throughout this article, denote the distance of the point $x \in \mathbb{R}^{n}$ to the origin by $r^{2}=|x|^{2}$. We shall prove that the integrals

$$
I_{m}(M):=\int_{M} r^{m} e^{-r^{2}} d \mu<\infty, \quad m=1,2, \ldots,
$$

are finite. We have

$$
I_{m}(M)=\sum_{j=0}^{\infty} \int_{M \cap\left(B_{j+1}-B_{j}\right)} r^{m} e^{-r^{2}} d \mu
$$

and consequently we can estimate $I_{m}(M)$ as follows:

$$
\begin{aligned}
I_{m}(M) & \leq \sum_{j=0}^{\infty}(j+1)^{m} e^{-j^{2}}\left[\operatorname{vol}\left(M \cap B_{j+1}\right)-\operatorname{vol}\left(M \cap B_{j}\right)\right] \\
& \leq \sum_{r=0}^{\infty}(r+1)^{m} e^{-r^{2}} \operatorname{vol}\left(M \cap B_{r+1}\right) .
\end{aligned}
$$

Using the assumption on the volume growth of $M$ we immediately obtain

$$
I_{m}(M) \leq C \sum_{r=0}^{\infty}(r+1)^{m+l} e^{-r^{2}}
$$

Denoting the summands of the latter series by $a_{r}$, we readily see that it converges, since

$$
\frac{a_{r+1}}{a_{r}}=\frac{(r+1)^{m+l} e^{-r^{2}-2 r-1}}{r^{m+l} e^{-r^{2}}}=\left(\frac{r+1}{r}\right)^{m+l} \frac{1}{e^{2 r+1}} \rightarrow 0 .
$$

A similar calculation yields the result for the functions $e^{\alpha r^{2}}$ with $\alpha<1 / 2$.

3. A dense subspace in $\mathcal{C}_{\infty}^{0}\left(S^{n}\right)$. The aim of this section is to verify that a certain linear subspace of $\mathcal{C}_{\infty}^{0}\left(S^{n}\right)$ is dense therein. Since the family of functions we have in mind cannot be made into an algebra, we have to replace the standard Stone-Weierstraß argument by something different. The idea for overcoming this problem is to use a combination of the wellknown theorems of Hahn-Banach, Riesz and Bochner. 
To begin with, we uniformly approximate the function $e^{-r^{2}} e^{i\langle k, x\rangle}$ for a fixed vector $k \in \mathbb{R}^{n}$.

Lemma 1. Denote by $p_{m}(x)$ the polynomial

$$
p_{m}(x)=\sum_{\alpha=0}^{m-1} i^{\alpha}\langle k, x\rangle^{\alpha} / \alpha ! .
$$

Then the sequence $e^{-r^{2}} p_{m}(x)$ converges uniformly to $e^{-r^{2}} e^{i\langle k, x\rangle}$ on $\mathbb{R}^{n}$.

Pr o of. The inequality

$$
\left|p_{m}(x)-e^{i\langle k, x\rangle}\right| \leq \frac{\|k\|^{m}\|x\|^{m}}{m !} e^{\|k\| \cdot\|x\|}
$$

implies (set $y=\|k\| \cdot\|x\|)$

$$
\sup _{x \in \mathbb{R}^{n}}\left|e^{-r^{2}} p_{m}(x)-e^{-r^{2}} e^{i\langle k, x\rangle}\right| \leq \sup _{0 \leq y} \frac{y^{m}}{m !} e^{y-y^{2} /\|k\|^{2}}=: C_{m} .
$$

Therefore, we have to check that for any fixed vector $k \in \mathbb{R}^{n}$ the sequence $C_{m}$ tends to zero as $m \rightarrow \infty$. For simplicity, denote by $k$ the length of the vector $k \in \mathbb{R}^{n}$. A direct calculation yields

$$
\begin{aligned}
C_{m}= & \frac{1}{m !}\left(\frac{k^{2}}{4}+\frac{k}{4} \sqrt{k^{2}+8 m}\right)^{m} \\
& \times \exp \left(\frac{k^{2}}{4}+\frac{k}{4} \sqrt{k^{2}+8 m}-\frac{1}{k^{2}}\left(\frac{k^{2}}{4}+\frac{k}{4} \sqrt{k^{2}+8 m}\right)^{2}\right) .
\end{aligned}
$$

We are only interested in the asymptotics of $C_{m}$. We will thus ignore all constant factors not depending on $m$. In this sense, we obtain

$$
C_{m} \approx \frac{1}{m !}\left(\frac{k^{2}}{4}+\frac{k}{4} \sqrt{k^{2}+8 m}\right)^{m} \exp \left(\frac{k}{8} \sqrt{k^{2}+8 m}-\frac{k^{2}+8 m}{16}\right) .
$$

The Stirling formula $m ! \approx \sqrt{m} m^{m} e^{-m}$ allows us to rewrite the asymptotics of $C_{m}$ :

$$
C_{m} \approx \frac{1}{\sqrt{m} m^{m}}\left(\frac{k^{2}}{4}+\frac{k}{4} \sqrt{k^{2}+8 m}\right)^{m} \exp \left(\frac{k}{8} \sqrt{k^{2}+8 m}+\frac{m}{2}\right) .
$$

Since

$$
\lim _{m \rightarrow \infty}\left(\sqrt{k^{2}+8 m}-\sqrt{8 m}\right)=0,
$$

we can furthermore replace $\sqrt{k^{2}+8 m}$ by $2 \sqrt{2 m}$ :

$$
C_{m} \approx \frac{1}{\sqrt{m} m^{m}}\left(\frac{k^{2}}{4}+\frac{k}{4} \sqrt{k^{2}+8 m}\right)^{m} \exp \left(\frac{k}{4} \sqrt{2 m}+\frac{m}{2}\right)=: e^{C_{m}^{*}}
$$

with

$$
C_{m}^{*}=m \ln \left(\frac{k^{2}}{4}+\frac{k}{4} \sqrt{k^{2}+8 m}\right)+\frac{k}{2 \sqrt{2}} \sqrt{m}+\frac{m}{2}-m \ln (m)-\frac{1}{2} \ln (m) .
$$


If $m$ is large compared with $k$, we can estimate $\ln \left(k^{2} / 4+(k / 4) \sqrt{k^{2}+8 m}\right)$ by $\frac{1}{2} \ln (m)+\alpha$ for some constant $\alpha$ :

$$
\begin{aligned}
C_{m}^{*} & \lesssim \frac{m}{2} \ln (m)+\alpha m+\frac{k}{2 \sqrt{2}} \sqrt{m}+\frac{m}{2}-m \ln (m)-\frac{1}{2} \ln (m) \\
& \leq-\frac{m}{2} \ln (m)+(\alpha+1 / 2) m+\frac{k}{2 \sqrt{2}} \sqrt{m} \\
& \leq-\frac{m}{2} \ln (m)+\left(\alpha+1 / 2+\frac{k}{2 \sqrt{2}}\right) m \\
& =m\left(\alpha+1 / 2+\frac{k}{2 \sqrt{2}}-\frac{1}{2} \ln (m)\right) .
\end{aligned}
$$

Finally, $C_{m}=\exp \left(C_{m}^{*}\right)$ converges to zero.

We denote the full ring of polynomials on $\mathbb{R}^{n}$ by $\mathcal{P}$.

Proposition 2. The linear space $\Sigma_{\infty}:=\mathcal{P} \cdot e^{-r^{2}}$ is dense in the space $\mathcal{C}_{\infty}^{0}\left(S^{n}\right)$ of all continuous functions on $S^{n}=\mathbb{R}^{n} \cup\{\infty\}$ vanishing at infinity.

Pr o of. Suppose the closure $\bar{\Sigma}_{\infty}$ of the linear space $\Sigma_{\infty}$ does not coincide with $\mathcal{C}_{\infty}^{0}\left(S^{n}\right)$. Then the Hahn-Banach Theorem implies the existence of a continuous linear functional $L: \mathcal{C}^{0}\left(S^{n}\right) \rightarrow \mathbb{R}$ such that

1. $\left.L\right|_{\Sigma_{\infty}}=0$;

2. $L\left(g_{0}\right) \neq 0$ for at least one $g_{0} \in \mathcal{C}_{\infty}^{0}\left(S^{n}\right)$.

According to Riesz' Theorem (see [Rud, Ch. 6, pp. 129 ff.]), $L$ may be represented by two regular Borel measures $\mu_{+}, \mu_{-}$on $S^{n}$ :

$$
L(f)=\int_{S^{n}} f(x) d \mu_{+}(x)-\int_{S^{n}} f(x) d \mu_{-}(x) .
$$

In particular, $\mu_{+}$and $\mu_{-}$are finite. The first property $\left.L\right|_{\Sigma_{\infty}}=0$ of $L$ implies

$$
\int_{S^{n}} e^{-r^{2}} p(x) d \mu_{+}(x)=\int_{S^{n}} e^{-r^{2}} p(x) d \mu_{-}(x)
$$

for any polynomial $p(x)$. Let us introduce the measures $\nu_{ \pm}=e^{-r^{2}} \mu_{ \pm}$on the subset $\mathbb{R}^{n} \subset S^{n}$. Then

$$
\int_{\mathbb{R}^{n}} p(x) d \nu_{+}(x)=\int_{\mathbb{R}^{n}} p(x) d \nu_{-}(x)
$$

holds and remains true for any complex-valued polynomial. We may thus choose $p(x)=p_{m}(x)$ as in the previous lemma:

$$
p_{m}(x)=\sum_{\alpha=0}^{m-1} i^{\alpha}\langle k, x\rangle^{\alpha} / \alpha !
$$


But then

$$
\begin{aligned}
\int_{S^{n}} p_{m}(x) e^{-r^{2}} d \mu_{+}(x) & =\int_{\mathbb{R}^{n}} p_{m}(x) d \nu_{+}(x)=\int_{\mathbb{R}^{n}} p_{m}(x) d \nu_{-}(x) \\
& =\int_{S^{n}} p_{m}(x) e^{-r^{2}} d \mu_{-}(x)
\end{aligned}
$$

together with the uniform convergence of $p_{m}(x) e^{-r^{2}}$ to $e^{i\langle k, x\rangle} e^{-r^{2}}$ implies

$$
\int_{S^{n}} e^{i\langle k, x\rangle} e^{-r^{2}} d \mu_{+}(x)=\int_{S^{n}} e^{i\langle k, x\rangle} e^{-r^{2}} d \mu_{-}(x),
$$

i.e.,

$$
\int_{\mathbb{R}^{n}} e^{i\langle k, x\rangle} d \nu_{+}(x)=\int_{\mathbb{R}^{n}} e^{i\langle k, x\rangle} d \nu_{-}(x) .
$$

Therefore, the Fourier transforms of the measures $\nu_{+}$and $\nu_{-}$coincide. Consequently, by Bochner's Theorem (see [Mau, Ch. XIX, pp. $774 \mathrm{ff}$.]) we conclude that $\nu_{+}=\nu_{-}$on $\mathbb{R}^{n}$. The linear functional $L: \mathcal{C}^{0}\left(S^{n}\right) \rightarrow \mathbb{R}$ must thus be the evaluation of a function at infinity:

$$
L(f)=c f(\infty),
$$

contrary to the existence of a function $g_{0} \in \mathcal{C}_{\infty}^{0}\left(S^{n}\right)$ satisfying $L\left(g_{0}\right) \neq 0$.

\section{The main result}

Theorem 1. Let the closed subset $M \subset \mathbb{R}^{n}$ be a smooth submanifold satisfying the polynomial volume growth condition. Then the ring $\mathbb{R}[M]$ of all polynomials on $M$ is a dense subspace of the Hilbert space $L^{2}\left(M, e^{-r^{2}} d \mu\right)$.

Proof. Consider the one-point compactification $\widehat{M} \subset S^{n}$ of $M \subset \mathbb{R}^{n}$. Then Tietze's Extension Lemma and Proposition 2 imply that

$$
\Sigma_{\infty}(\widehat{M}):=\mathbb{R}[M] \cdot e^{-r^{2} / 4}
$$

is dense in $\mathcal{C}_{\infty}^{0}(\widehat{M})$. We introduce the measure $d \nu=e^{-r^{2} / 2} d \mu$, where $d \mu$ is the volume form of $M$. Since

$$
\int_{M} d \nu=\int_{M} e^{-r^{2} / 2} d \mu=\int_{M}\left(e^{r^{2} / 4}\right)^{2} e^{-r^{2}} d \mu=: V<\infty,
$$

$d \nu$ defines a regular Borel measure $d \widehat{\nu}$ on $\widehat{M}$ (by setting $d \widehat{\nu}(\infty)=0$ ). Therefore, the algebra $\mathcal{C}_{\infty}^{0}(\widehat{M})$ of all continuous functions on $\widehat{M}$ vanishing at infinity is dense in $L^{2}(\widehat{M}, d \widehat{\nu})$ :

$$
\overline{\mathcal{C}_{\infty}^{0}(\widehat{M})}=L^{2}(\widehat{M}, d \widehat{\nu}) .
$$


For any function $f$ in $L^{2}\left(M, e^{-r^{2}} d \mu\right)$ we have

$$
\int_{M}\left|f e^{-r^{2} / 4}\right|^{2} e^{-r^{2} / 2} d \mu=\int_{M}|f|^{2} e^{-r^{2}} d \mu<\infty
$$

and, therefore, $f e^{-r^{2} / 4}$ lies in $L^{2}(\widehat{M}, d \widehat{\nu})$. Thus, for a fixed $\varepsilon>0$, there exists $g \in \mathcal{C}_{\infty}^{0}(\widehat{M})$ such that

$$
\int_{M}\left|f e^{-r^{2} / 4}-g(x)\right|^{2} e^{-r^{2} / 2} d \mu<\varepsilon / 2 .
$$

According to Proposition 2 we can find a polynomial $p(x) \in \mathbb{R}[M]$ approximating $g$ :

$$
\sup _{x \in \widehat{M}}\left|g(x)-p(x) e^{-r^{2} / 4}\right|^{2}<\varepsilon /(2 V) .
$$

Using the inequality $\|x+y\|^{2} \leq 2\|x\|^{2}+2\|y\|^{2}$ we conclude that

$$
\int_{M}\left|f(x) e^{-r^{2} / 4}-p(x) e^{-r^{2} / 4}\right|^{2} e^{-r^{2} / 2} d \mu<\varepsilon ;
$$

but this is equivalent to

$$
\int_{M}|f(x)-p(x)|^{2} e^{-r^{2}} d \mu<\varepsilon .
$$

REMARK 1. In fact, the smoothness of $M$ is not essential in the proof of Theorem 1. By the same arguments, the main result holds for any manifold $M$ closed in $\mathbb{R}^{n}$, provided that $M$ admits a volume form such that the condition of polynomial volume growth as formulated in Proposition 1 is satisfied.

5. Examples and final remarks. We now give a few simple examples. Notice that we recover, of course, that the polynomials are dense in $L^{2}\left(\mathbb{R}^{n}, e^{-r^{2}} d \mu\right)$ (Hermite polynomials) or in $L^{2}(M, d \mu)$ for any compact submanifold (Legendre polynomials in the case $M=[-1,1]$ ).

EXAmple 1 . Consider a revolution surface in $\mathbb{R}^{3}$ defined by two polynomials $f, h$,

$$
\left\{\begin{array}{l}
x=f\left(u_{1}\right) \cos u_{2}, \\
y=f\left(u_{1}\right) \sin u_{2}, \quad f\left(u_{1}\right)>0,\left(u_{1}, u_{2}\right) \in \mathbb{R} \times[0,2 \pi] . \\
z=h\left(u_{1}\right),
\end{array}\right.
$$

Then $d \mu=f \sqrt{f^{\prime 2}+h^{\prime 2}} d u_{1} d u_{2}$ and $r^{2}=f^{2}+h^{2}$, and thus

$\mathbb{R}\left[f \cos u_{2}, f \sin u_{2}, h\right]$ is dense in

$$
L^{2}\left(\mathbb{R} \times[0,2 \pi], e^{-\left(f^{2}+h^{2}\right)} f \sqrt{f^{\prime 2}+h^{\prime 2}} d u_{1} d u_{2}\right) .
$$


In the special case of a cylinder, i.e. $f=1, h=u_{1}$, this reduces to the well known fact that the ring

$$
\mathbb{R}\left[u_{1}, \cos u_{2}, \sin u_{2}\right]=\mathbb{R}\left[u_{1}\right] \otimes \mathbb{R}\left[\cos u_{2}, \sin u_{2}\right]
$$

is indeed dense in the Hilbert space

$$
L^{2}\left(\mathbb{R} \times[0,2 \pi], e^{-u_{1}^{2}} d u_{1} d u_{2}\right)=L^{2}\left(\mathbb{R}, e^{-u_{1}^{2}} d u_{1}\right) \otimes L^{2}\left([0,2 \pi], d u_{2}\right) .
$$

Example 2. Let $F: \mathbb{C} \rightarrow \mathbb{C}$ be a polynomial and consider the surface defined by

$$
f: \mathbb{C} \rightarrow \mathbb{R}^{3}, \quad f(z)=(x, y,|F(z)|), \quad z=x+i y .
$$

Then one checks that $d \mu=\sqrt{1+\left|F^{\prime}\right|^{2}}|d z|^{2}$ and $r^{2}=|z|^{2}+|F(z)|^{2}$. Thus

$$
\overline{\mathbb{R}[x, y,|F(z)|]}=L^{2}\left(\mathbb{R}^{2}, e^{-\left(|z|^{2}+|F(z)|^{2}\right)} \sqrt{1+\left|F^{\prime}\right|^{2}}|d z|^{2}\right) .
$$

Let us study the polynomial $F=z^{2 k}$ in more detail. Here the coordinate ring coincides with the usual polynomial ring $\mathbb{R}[x, y]$ in two variables, and thus we have proved that these are dense in

$$
L^{2}\left(\mathbb{R}^{2}, e^{-\left(|z|^{2}+|z|^{4 k}\right)} \sqrt{1+4 k^{2}|z|^{2(2 k-1)}}|d z|^{2}\right) .
$$

EXAMPLE 3. We finish with a one-dimensional example: the graph $M=$ $\{(x, f(x))\}$ of a polynomial $f: \mathbb{R} \rightarrow \mathbb{R}^{n}$. Then $d \mu=\sqrt{1+\left\|f^{\prime}\right\|^{2}} d x$, and we obtain

$$
\overline{\mathbb{R}[x]}=L^{2}\left(\mathbb{R}, e^{-\left(x^{2}+\|f(x)\|^{2}\right)} \sqrt{1+\left\|f^{\prime}\right\|^{2}} d x\right) .
$$

REMARK 2. The main result raises an interesting analogous problem in complex analysis which, to our knowledge, is still open. It is well known that the polynomials on $\mathbb{C}^{n}$ are dense in the Fock or Bergman space

$$
\mathcal{F}\left(\mathbb{C}^{n}\right):=\left\{f \in L^{2}\left(\mathbb{C}^{n}, e^{-r^{2}} d \mu\right) \mid f \text { holomorphic }\right\} .
$$

Furthermore, a theorem by Stoll (see [Sto1], [Sto2]) states that among all complex-analytic submanifolds $N$ of $\mathbb{C}^{n}$, those with polynomial growth are exactly the algebraic ones, and thus the only ones for which the elements of the coordinate ring are square-integrable with respect to the Gaussian measure. It is then common to study the space

$$
\mathcal{F}(N):=\left\{f \in L^{2}\left(N, e^{-r^{2}} d \mu\right) \mid f \text { holomorphic }\right\},
$$

but we were not able to find any results on whether $\mathbb{C}[N]$ is dense herein.

More elaborate applications of the main result to the situation where $M$ carries a reductive algebraic group action will be discussed in some forthcoming works (see e.g. $[\mathrm{Agr}]$ ). In this case, one can decompose the ring $\mathbb{R}[M]$ into isotypic components and, via Theorem 1 , one obtains a decomposition of $L^{2}\left(M, e^{-r^{2}} d \mu\right)$ analogous to the classical Frobenius reciprocity. 


\section{References}

[Agr] I. Agricola, Dissertation am Institut für Reine Mathematik der HumboldtUniversität zu Berlin, in preparation.

[Brö] L. Bröcker, Semialgebraische Geometrie, Jahresber. Deutsch. Math.-Verein. 97 (1995), 130-156.

[Mau] K. Maurin, Analysis, Vol. 2, Reidel and PWN-Polish Sci. Publ., Dordrecht and Warszawa, 1980.

[Mi1] J. Milnor, On the Betti numbers of real varieties, Proc. Amer. Math. Soc. 15 (1964), 275-280.

[Mi2] -, Euler characteristics and finitely additive Steiner measures, in: Collected Papers, Vol. 1, Publish or Perish, 1994, 213-234.

[Rud] W. Rudin, Real and Complex Analysis, McGraw-Hill, 1966.

[Sto1] W. Stoll, The growth of the area of a transcendental analytic set. I, Math. Ann. 156 (1964), 47-78.

[Sto2] -, The growth of the area of a transcendental analytic set. II, ibid. 156 (1964), 144-170.

Institut für Reine Mathematik

Humboldt-Universität zu Berlin

Ziegelstr. $13 \mathrm{~A}$

D-10099 Berlin, Germany

E-mail: agricola@mathematik.hu-berlin.de friedric@mathematik.hu-berlin.de 